Proceedings of the 2011 Winter Simulation Conference

S. Jain, R.R. Creasey, J. Himmelspach, K.P. White, and M. Fu, eds.

\title{
UNDERSTANDING THE IMPACT OF COMMUNICATIONS TECHNOLOGIES ON VIRTUAL TEAM PERFORMANCE: AN AGENT-BASED SIMULATION MODEL
}

\author{
Vikas Sahasrabudhe \\ The George Washington University \\ 2201 G Street NW, Suite 515 \\ Washington, DC 20052, USA
}

\author{
Shivraj Kanungo \\ The George Washington University \\ 2201 G Street NW, Suite 415 \\ Washington, DC 20052, USA
}

\author{
Ramakrishna Iyer \\ The George Washington University \\ 2201 G Street NW, Suite 515 \\ Washington, DC 20052, USA
}

\begin{abstract}
Enterprises are constantly looking for ways to get the most from their geographically dispersed human resources by forming virtual teams, and leveraging communications technologies for enabling good team performance. The experience in using these technologies by virtual teams has been mixed at best, and the extant literature has gaps in offering satisfactory explanation for the variations. To address that gap, we have developed an agent-based simulation model to understand the dynamic complexities of the interplay between the characteristics of a virtual team, the task of the team, individuals forming the team, and the key functionalities provided by communications technologies, and to simulate the collaboration and work done by the team for its assigned tasks. Preliminary results point to the potential usefulness of the model to investigate the impact of communications technologies on virtual team performance.
\end{abstract}

\section{INTRODUCTION}

We study the role of communications technologies in teams that require collaboration among their members for satisfactory performance of their functions. The backdrop for this study is the emergence and rapid evolution of communications technologies that allow users to interact almost as if they were face-toface (e.g., virtual presence, multimedia, etc.). Specifically, the growing maturity of Internet Protocol (IP) based delivery of interactive voice, data and video services has triggered significant technological developments in the design and delivery of new "collaboration" oriented Unified Communications (UC) services to end-users. UC is currently in early stages of introduction and it has so far been a technology "push" primarily by vendors and service providers. However, how this technology will be used and how it will spread is unclear. Our study is motivated by a need to develop a better understanding of its applicability and use. The UC environment of the future may potentially result in distance interactions that are richer than the types of interactions today. With the growing emphasis on telecommuting employees, the UC work environment will be important for telecommuters in the future. However, Gartner Group (2008) mentioned that models for the adoption of these new services by end-users in an organizational context have not been studied extensively.

The primary contribution of our study lies in the development of a theoretical model that initiates the exploration of the comparison between virtual collaboration (i.e., using communications technologies) 


\section{Sahasrabudhe, Kanungo, and Iyer}

and face-to-face collaboration (implying primarily physical proximity, typically being in the same room) in order to better understand the impact of these collaboration modes on organizational performance. We have operationalized the theoretical model using agent-based simulation technique since the theoretical model does not easily lend itself to other types of modeling using field or laboratory settings.

\section{BACKGROUND}

Over the years, a significant amount of research has been conducted on individual "technology acceptance" in the context of information systems. Most such studies have been based on evaluating users' perception of ease of use and usefulness associated with information technologies. Relatively less research attention has been placed on analyzing the impact of new technologies on processes at various levels within enterprises. While the use of communications technologies for collaboration among members in organizations has received significant attention from vendors of technologies and services, there have been very few academic studies directed at understanding the dynamic complexities of the use of such technologies in organizations. The few studies that have addressed the problem have done so from different standpoints that are narrowly focused. In order to illustrate the variety of past approaches, we present a summary of a limited set of past studies.

- Guthrie, Rosen, and Salas (2007) provide a comprehensive theoretical overview of the setting where technology based collaboration is framed as an interaction between team level constructs and individual constructs. The dependent variable (team effectiveness) is shown to feed back into team level constructs.

- Goodhue and Thompson (1995) provide guidance on the fit between the nature of the task and information technology in use. While theirs is a generic approach to technology acceptance, it informs us importantly that communications technologies have to be relevant or appropriate to the nature of the task or interaction - even though their model is a static one.

- Walsh and Maloney (2007) conducted four field experiments on the use of e-mail (in experimental biology, mathematics, physics and sociology) and highlighted the problems of coordination and misunderstanding, of cultural differences and of information security. However, their survey based study was only for use of email.

- Jarvenpaa and Lang (2005) conducted a qualitative study of mobile device users and focused on user-technology interactions. They studied purposes of technology use, situations factors and coping strategies. Their unique insights highlight the presence of paradoxes in the sense that the very technologies that encourage the user to use them also end up being the source of user dissatisfaction when their use goes beyond a certain threshold.

- Volkoff, Strong, and Elmes (2007) employ a qualitative study to theorize the relationship between technology, human agency and organizational change, and in doing so employ the critical realist perspective. Their model explains how the process unfolds, identifying the mechanisms that move it along. Their observation that the introduction of a technology is associated with multiple contingencies acting at multiple levels and that it is a major challenge to predict this emergence as this process unfolds is particularly relevant for our study.

A major challenge from a theoretical perspective is in incorporating the interaction, over time, between team characteristics, individual characteristics and the nature of the task(s) in determining the effectiveness of any communications technology. From a practical standpoint, determining the benefits of such technologies, and the mechanisms and managerial levers for steering such initiatives toward meaningful organizational results is equally challenging. Our study addresses some of these challenges by recognizing the complexities and developing a model to address them. The model we present here is viewed as an initial step in the representation and assessment of some of the complexities, and enhancements to the model can be made in a number of areas. However, the attempt is to create the basic formulations that could then be further developed to capture the more complex interactions. In the next section we describe our theoretical model and highlight the main literature streams that have guided the development of the theoretical model. 


\section{THEORETICAL MODEL}

Past research in the area of technology use (specifically information technology) has been an important guide for our work. We use behavioral perspective in the context of using communications technologies for collaboration because our objective was to study how the set of influences between and across individuals and teams plays out in determining team performance. Our theoretical model is based broadly on the IMO model (Mathieu et al., 2008) shown in Figure 1.

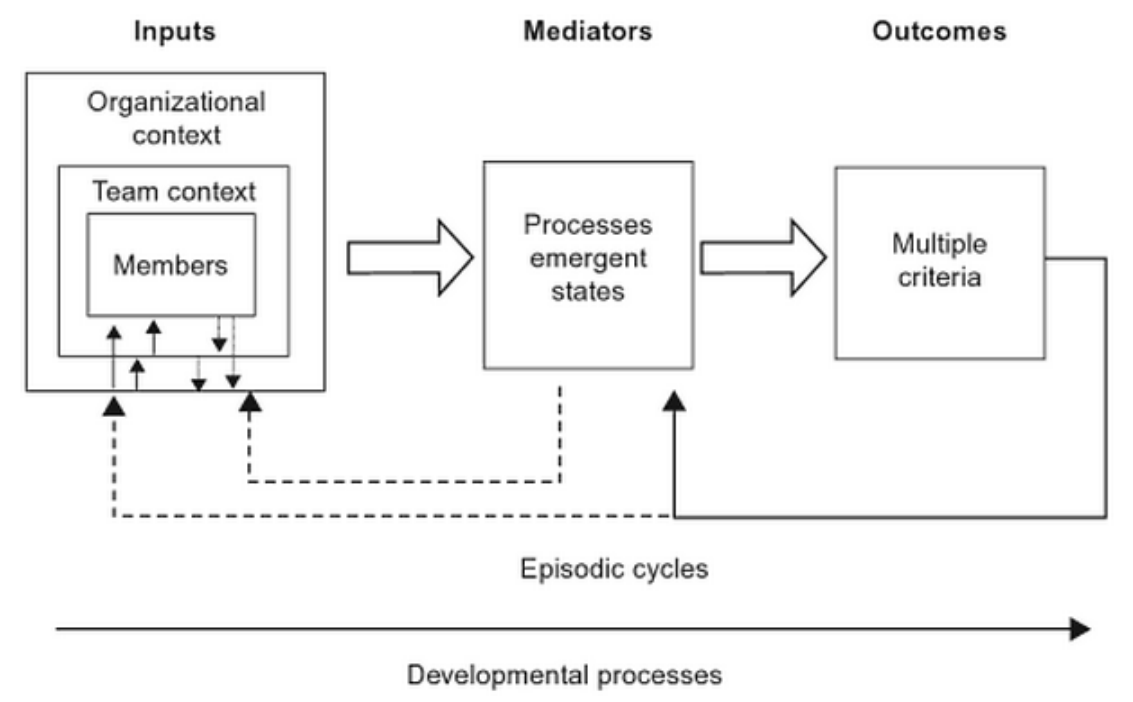

Figure 1: Input-Mediator-Outcome (IMO) Team Effectiveness Framework

We begin by postulating that organizational entities require different levels of collaboration for satisfactory performance of their tasks or functions, and that there is an optimal level of collaboration (referred to as ECTask) for an organizational entity to achieve peak performance. During a performance period, when the actual collaboration within the organizational entity matches this optimum value, the entity performs at its peak and the performance drops off whenever the actual collaboration deviates from the optimum value. In this context, collaboration above the optimum value is as bad as collaboration below the optimum value. Note that while we use the term organizational entity above, it could equivalently represent a smaller subset of a larger organization that has a well-defined function, or it could even be a task oriented project team. We will use the terms "team" and "task" in the rest of this paper with this implicit extended meaning.

Using the definition of "team" and "task" mentioned above, we apply the IMO model as a framework to analyze the collaboration process of a team with and without mediating technology. Since communications technologies are for sharing information and not for any physical activities, our model is focused on intellectual work teams.

Many published studies have shown that the extent of collaboration among team members is dependent on factors such as team composition, trust between team members, and learning by individuals and the team (Stewart 2006; Curseu 2006; Matheu et al. 2008; Miller and Lin 2010; Steinfeld et al. 2001). We have included these factors in our model. Similarly, Kozlowski and Ilgen (2006) have noted the importance of task characteristics, and Zhang, Fjermestad, and Tremaine (2005) have reported that communication media richness has an impact on the work of teams. In our model, we have also included these two components.

A number of papers have reported on the influence of leadership, particularly leadership style, on the work output of teams (Hambley, O'Neill, and Kline 2007; Balthazard et al. 2004; Burke et al. 2006). We have made a provision for including leadership style as a parameter in our model. However, the effect of varying that parameter is not covered in the results presented in this paper. 
Using the published results mentioned above, we have simplified the general IMO model by focusing on key variables pertaining to collaborative performance of teams. From the perspective of inputs, one essential component of the input is the team itself, namely the collaborative characteristics of the members in the team as well as the motivation of the team to perform its function/task. Other inputs are the optimum collaborative requirement to perform the function/task effectively and the characteristics of the communications technology employed by the organization to facilitate collaboration among its members. The emergent states in our model would primarily relate to the evolution of the characteristics of the team and its members, since the team task or function as well as technology components are assumed to be unchanged during the observation interval. The main outputs are the extent of collaboration during each performance period and the work output generated by the team, and these outputs in turn affect the inputs as well as the emergent states. Figure 2 shows our theoretical model, derived from the IMO model, for the purposes of our study.

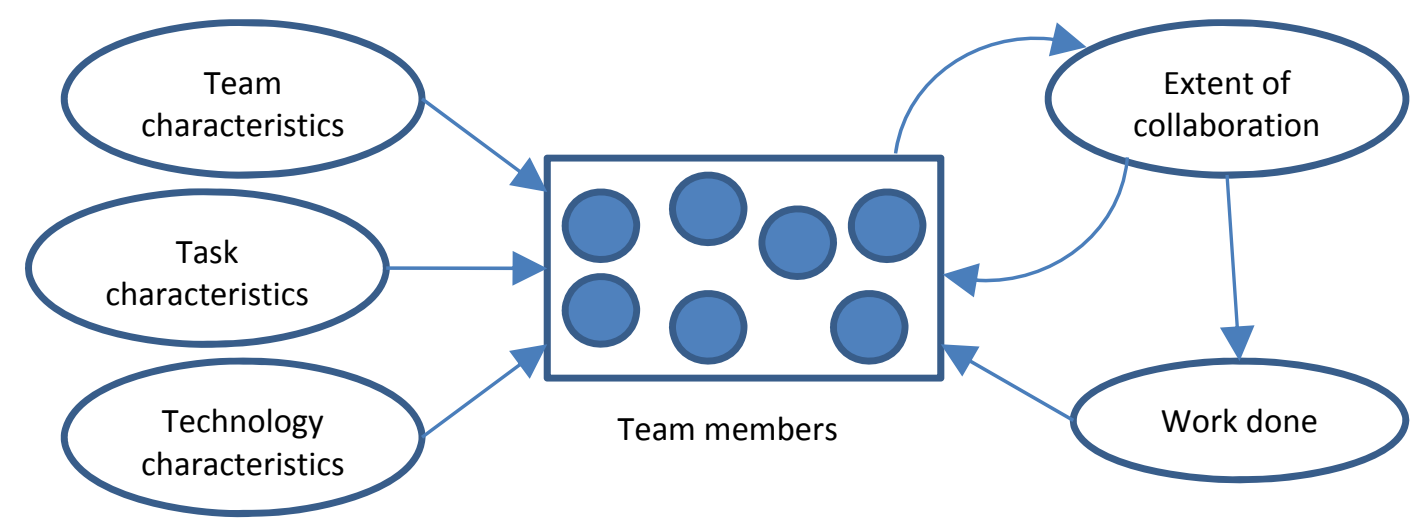

Figure 2: Simplified Collaboration Model for Teams

As indicated earlier, in our theoretical model, we define two modes of collaboration - face-to-face and virtual. Individuals in the team are assumed to have varying collaborative abilities, and this is reflected as one of the team input parameter in the model. The individual's collaborative ability is dependent on the mode of collaboration. For example, an individual may have a high ability to collaborate via face-to-face, but may lack the ability when using communications technology. In most practical/realistic cases, a team would consist of a heterogeneous combination of individuals with varying levels of collaborative abilities.

We introduce the concept of a task "collaboration index" that defines the collaboration level that must be achieved to best carry out the team's task/function. Measures for the characterization of this index are still under development. The notion introduced in our theoretical model is that, if the actual collaboration between members of the team matches this index, the productivity of the team will be a maximum. When the actual collaboration departs from this index, the observed productivity will drop off linearly. Note that collaboration in excess of the task's "collaboration index" has the same effect on loss of productivity as collaboration below the index.

The collaboration abilities of individuals in the organization will determine their initial productive collaboration effort (or coefficient) which will change from one time period to another. This corresponds to the "emergent states" of the team during the performance of the group task. Individuals in the group will tend to gravitate their productive collaboration coefficient to the team average over time. The influence of work outcome, however, will depend on the motivation level of the individual. Individuals with high motivation will tend to be more affected by negative work outcome than individuals with low motivation. The above influencers impact both face-to-face and virtual productive collaboration coefficients in a similar manner. The third influencer, that impacts only the virtual collaboration coefficient, has to do with technological efficacy of the individual. While each individual may have a different "technological efficacy" at the beginning, over time they may show an improvement of the efficacy and demonstrate a 


\section{Sahasrabudhe, Kanungo, and Iyer}

higher level of familiarity and comfort with using the underlying technologies in the virtual communications environment if there is sufficient collaboration in that mode.

Finally, in our theoretical model, we postulate that the face-to-face collaboration achieved by a pair of team members during a performance period is directly dependent on the collaboration abilities of the individuals. However, for the virtual environment, the communications technology will also have a strong influence on the collaboration between the individuals. In our model, we conceptualize the multiple facets of communications technologies and their impact on collaboration between individuals by a technology multiplier (TM) factor. In general, this will be a multidimensional entity, (a vector representation would be appropriate) but for the purposes of this initial model we represent it as a single dimensional variable. TM represents the relative impact of communications technologies on collaboration compared to face-toface collaboration among team members without any communications technology. A value of TM of less than one denotes that technology based collaborative techniques are not as effective as face-to-face meetings. It must be noted that the communications technologies currently deployed in most organizations will have a TM value of less than one (Olson and Olson, 2000), but with the introduction of more sophisticated UC communications applications this value could be greater than one as noted by Olson and Olson in their reference. Measures and methodology for characterization of TM is being explored in parallel to this effort.

Based on the model described above, we expect that introducing communications technology that has high technology multiplier (TM) may increase team performance in many cases but not all. Team composition, team motivation and task characteristics will influence that relationship.

\section{SIMULATION MODEL FORMALIZATION}

Our theoretical model has the characteristics of a complex evolving system. Of the ten principles of complexity, articulated in Mittleton-Kelly (2003, Ch. 2, 23-50), our model clearly includes:

- Connectivity and interdependence among team members, team, task and technology

- Feedback every time period from the extent of collaboration and work done on team member attributes

- Emergence of a new state or order that will be applicable to the subsequent time period for performance of the task by the team members

Given the complexities of the theoretical model, we decided to formalize a simulation model. We selected an agent-based simulation model because collaboration is an inherently group-based phenomenon where one team member's actions depend on, and have an impact on each of the other team members. Collaboration, whether face-to-face or via technology, has to work within the context of the organization but cannot be dictated by the organization. Uses of agent-based modeling for similar intellectual processes have appeared in the literature. Canessa and Riolo (2006) presented an agent-based model of the impact of computer-mediated communication on organizational culture and performance. Mollona and Jesi (2008) described their agent-based model to study knowledge integration in large organizations. Takahashi (2006) adopted agent-based modeling approach for organizational learning. In fact, agentbased modeling technique is used extensively in organizational science to study culture, social systems, dynamic team formation, and emergent network structure (Gautam, Singh, and Singh 2009; Gaston and des-Jardins 2005; Hazy and Tivnan 2004; Tivnan, 2005).

In our agent-based model, each agent represents a member of a team that is working on a task that extends over several time periods. We define collaboration and productivity attributes for each team member. The autonomous as well as team dependent behavior of each member is strongly influenced by the value of these attributes. The collaborative behavior of every agent pair in the team is computed for each time period taking into account the specific attributes of the pair. Furthermore, the impact of the collaboration and productivity during that period on agent attributes is also evaluated to define the emergent states of the team after every time period. In formulating our agent based model, we used some of the ideas that have been used in other social simulation models from the NetLogo model library, e.g., modeling 
of spread of contagion, modeling of cooperation and altruism in groups, and modeling of team assembly behavior.

To formalize our agent-based model, we start by defining a set of attributes for each team member (agent). Specifically, each agent starts with a productive collaboration coefficient for face to face interaction (PCF), productive collaboration coefficient for interaction via technology (PCT), self-efficacy in the specific technology to be used (SE), productivity in terms of maximum work units completed per time period (PR), motivation to get the work done up to his/her full productivity (MF), and perceived usefulness of the technology (PUT). Members in a team may have different PCF and PCT. Trust among each pair depends on the initial PCF of each member of that pair.

As included in our theoretical model, the specific function/task to be carried out by the organization/team requires a certain optimum level of collaboration (ECTask). The technology chosen will (based on its characteristics) provide a certain multiplier effect for collaboration (TM). Our model includes a provision to define a threshold for PUT that is considered equivalent to face-to-face collaboration in the team, and a threshold for technology-enabled collaboration below which a team member will not learn enough about the technology to increase his/her self-efficacy. The model also provides for defining coefficients for rate of change in self-efficacy and in productive collaboration coefficient. The output variables of interest in the model are work done face-to-face (WDF), and work done via technology (WDT).

\section{AGENT BASED MODEL IMPLEMENTATION}

We implemented the model in NetLogo software with an interface (Figure 3) that allows us to set the team variables (team composition, average team motivation), task variable ECTask, TM for the technology being considered, and the number of time periods (each time period was assumed to be a week) for the simulation run.
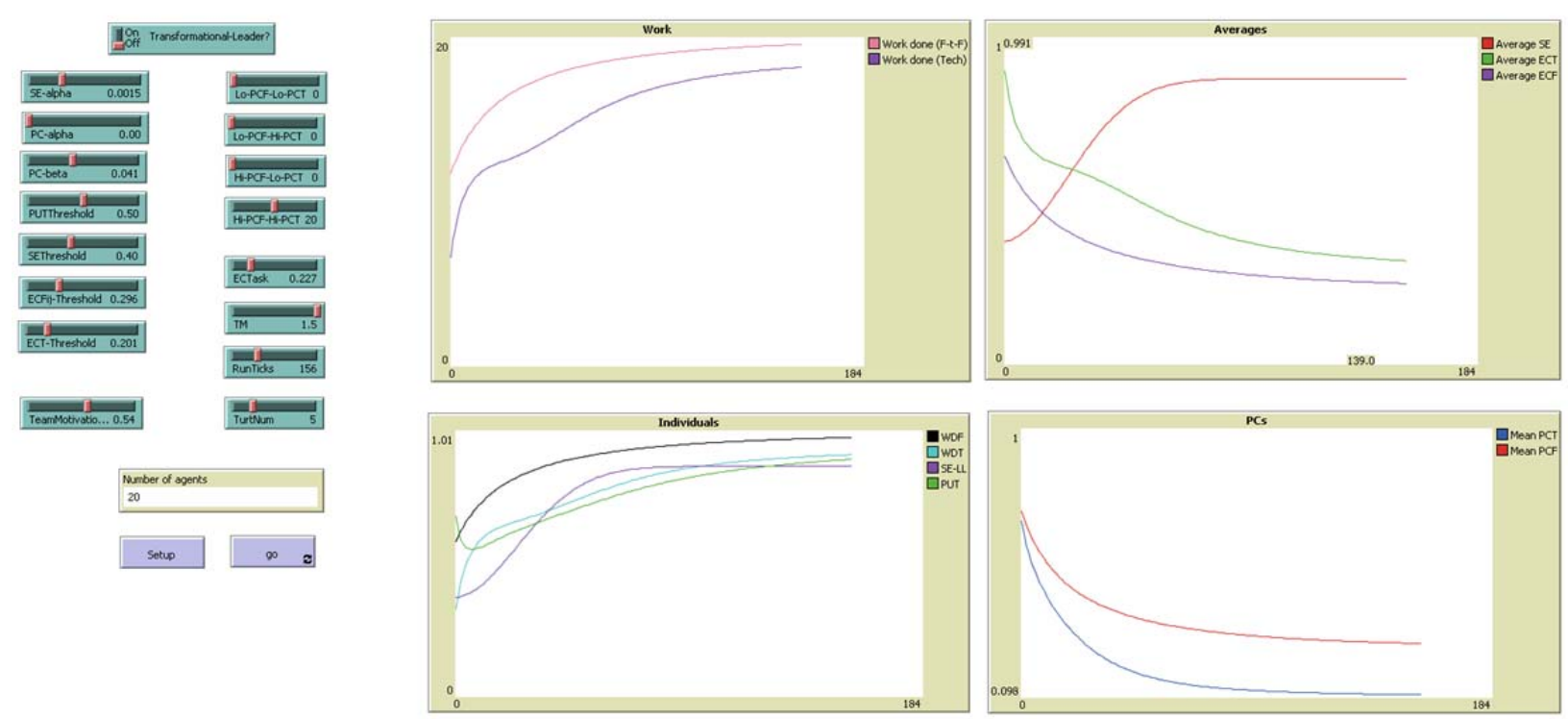

Figure 3: Agent-based Model interface in NetLogo

In the implementation of our agent-based model, we start with the team composition in terms of members' collaboration abilities. The model first initializes the following variables for each team member - PCF, PCT, MF (random around the team average), and self-efficacy. PUT for each team member is set randomly around the initial value of PCT. The trust among each pair of team members is initialized based on the initial PCF of those two members. We have assigned numerical values in the range of $0-1$ for most of the parameters to reflect the variability of the characteristics. For each time period, the model 


\section{Sahasrabudhe, Kanungo, and Iyer}

calculates the extent of collaboration and work done by each team member and the team using the following formulations:

- $E C F_{i j}=\sqrt{P C F_{i} \times P C F_{j}} \times$ Trust $_{i j}$ Extent of collaboration (ECF) face-to-face by a pair of team members is based on the geometric mean of PCF for the pair weighted by the trust between the pair. We employ the geometric mean to capture the attenuation of collaboration of pairs with low ECFs and the high degree of collaboration of pairs with high ECFs. This is weighted by the degree of trust between the pairs of collaborating individuals.

- $E C F_{i}=\sum_{j=1, i \neq j}^{n} E C F_{i j}$ Extent of collaboration by a member is the sum of ECF with all other members of the team. This is a simple sum of the individual's extent of collaboration with everyone else.

- $W D F_{i}=P R \times\left(1-\left|E C F_{i}-E C T a s k\right|\right)$ Work done face-to-face by a member is a function of the individual's productivity (PR) and level of collaboration. We assume that the appropriate level of collaboration (not too high and not too low, determined by the optimum level of collaboration (ECTask)) will maximize the work done. This happens when an individual's ECF is the same as the optimum level of collaboration.

- $\quad W D F_{\text {Team }}=\sum_{i=1}^{n} W D F_{i}$ Work done face-to-face by the team is the sum of work done face-toface by each member. The total work of the team is a simple sum, i.e., we do not assume any overheads or synergy across individuals.

- $E C T_{i j}=\sqrt{P C T_{i} \times P C T_{j}} \times \frac{S E_{i}+S E_{j}}{2} \times$ Trust $_{i j} \times T M$ Extent of collaboration via technology (ECT) by a pair of team members is based on the geometric mean of PCT for the pair, the arithmetic mean of SE for the pair, trust of the pair, and a technology multiplier (TM). This is conceptually identical to $E C F_{i j}$ with additional consideration for the collaborating pairs' technology selfefficacy and sophistication of the collaboration technology.

- $E C T_{i}=\sum_{j=1, i \neq j}^{n} E C T_{i j}$ Extent of technology based collaboration by a member is the sum of ECT with all other members of the team. This is a simple sum of the individual's extent of technology based-collaboration with everyone else.

- $W D T_{i}=P R \times\left(1-\mid E C T_{i}-E C T\right.$ ask $\left.\mid\right)$ Similar to $\mathrm{WDF}_{\mathrm{i}}$, we assume that the appropriate level of technology-based collaboration (not too high and not too low, determined by the optimum level of collaboration (ECTask)) will maximize the work done via technology. This happens when an individual's ECT is the same as the optimum level of collaboration.

- $W D T_{\text {Team }}=\sum_{i=1}^{n} W D T_{i}$ Work done via technology by the team is the sum of work done via technology by each member. As in the face to face case, we do not assume any overheads or synergy across individuals in case of work done via technology.

The model then determines, for each time period, the changes in the characteristics of each team member that will be used in the subsequent time period. PCF of each team member changes asymptotically towards a high of 0.9 or towards a low of 0.1 depending on the driver for change that is based on the mean PCF for the team and the gap in work done face-to-face compared to productivity. We decided to use lower and upper limits because realistically no one is totally against collaboration or totally for collaboration. Similarly, PCT of each team member changes asymptotically toward a high of 0.9 or toward a low of 0.1 depending on the driver for change that is based on the mean PCT for the team, the gap in work done via technology compared to productivity, and the perceived usefulness of the technology compared to a threshold for the team/organization. Self-efficacy (SE) increases asymptotically up to an upper limit for every time period when the member uses the technology effectively. Perceived usefulness of the technology changes asymptotically to a high of 0.9 or a low of PUT/PR depending on the driver for change that is based on work done via technology compared to the work done face-to-face. The equations used in the model to calculate these changes are given below. 
- $\quad$ PCF change driver $=$ PCMultiplier $*($ mean $[\mathrm{PCF}]$ of team members $-\mathrm{PCF}) \pm(\mathrm{MF} *$ WGAPF $))$

- $\quad$ PCT change driver $=$ PCMultiplier $*($ mean $[\mathrm{PCT}]$ of team members $-\mathrm{PCT}) \pm(\mathrm{MF} *$ WGAPT $)) *$ (PUT / PUTThreshold)

- $\quad$ SE change driver $=$ SE-alpha $*$ Coll-Ticks

- $\quad$ PUT change driver $=((\mathrm{WDT}-\mathrm{WDF}) /(\mathrm{WDF}+\mathrm{WDT}))$

The model implementation provided the capability to run experiments consisting of multiple simulation runs for the same set of agent attributes and team parameters. This process eliminates bias in the simulation due to a single dominant parameter. The experimental results were exported to Excel for developing the charts presented in the next section.

\section{SIMULATION RESULTS AND OBSERVATIONS}

To obtain insight into the potential consequences of introducing communications technology with specific collaboration functionalities in the context of a team, we ran the model for four scenarios for specific team settings. For these runs, the contextual parameters were set as follows: team size of 20, maximum work possible by each team member (PR) of 1, PC-beta 0.04, SE-alpha 0.0015, SE-threshold 0.4, PUTthreshold 0.5. These parametric values were arrived at after several trial runs to ensure that the simulation was not biased by a single dominant parameter. In each scenario, low TM was set at 0.5 and high TM at 1.5 .

Scenario 1 is where a task requiring low collaboration (ECTask 0.05 ) is assigned to a poorly motivated (motivation 0.25 ) heterogeneous team, i.e., a team with members distributed equally in terms of initial productive collaboration coefficients PCF of 0.25 and 0.75 , PCT of 0.25 and 0.75 . The results of this scenario, given in Figure 4, show that if the task requires low collaboration, technology with low TM will give higher productivity than face-to-face interaction for a heterogeneous team with low motivation. For that team for that task, technology with high TM will give somewhat lower productivity than face-to-face interaction. The collaboration benefits offered by enhanced communications technologies do not help in the performance of the task since it inherently does not require collaboration.

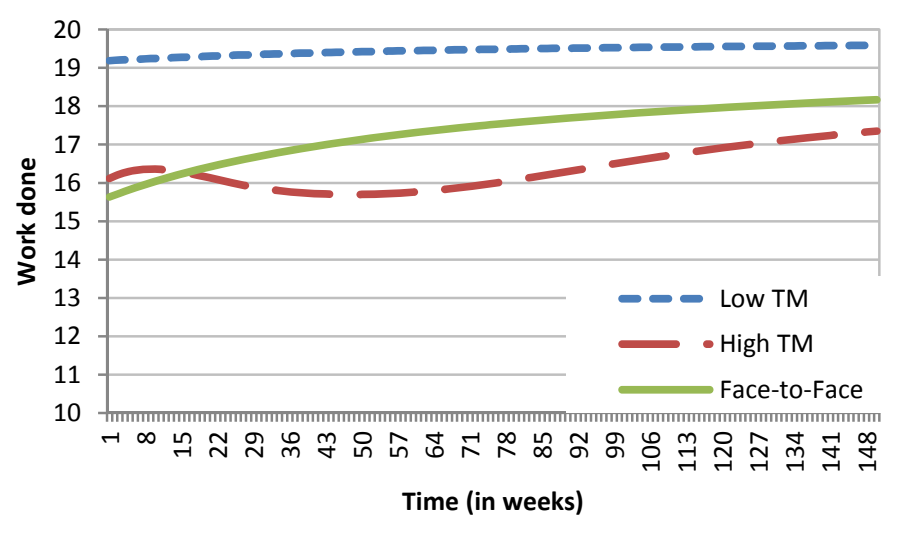

Figure 4: Scenario 1 results

Scenario 2 is where a task requiring high collaboration (ECTask 0.5 ) is assigned to a highly motivated (motivation 0.75 ) heterogeneous team, i.e., team with members distributed equally, as in scenario 1 , in terms of initial productive collaboration coefficients PCF of 0.25 and 0.75 , and PCT of 0.25 and 0.75 . The results of this scenario, given in Figure 5, show that for a heterogeneous team with high motivation, technology with high TM gives higher productivity than that from face-to-face interactions in the later time periods. Collaboration benefits offered by enhanced communications technologies are of value in 
this situation. Conversely, conventional communications technologies with low TM have a negative impact and result in decidedly low productivity.

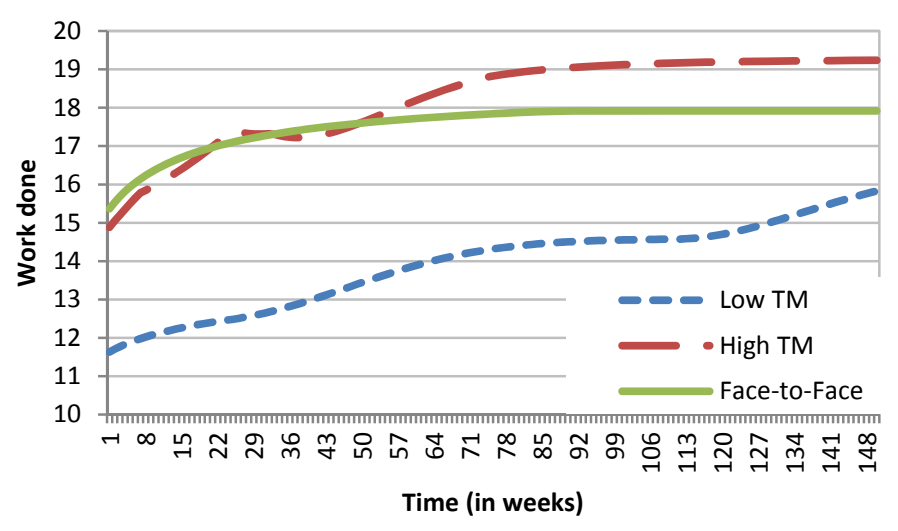

Figure 5: Scenario 2 results

Scenario 3 is where a task requiring high collaboration (ECTask 0.5) is assigned to a poorly motivated (motivation 0.25 ) homogeneous low collaboration team, i.e., all team members have low initial productive collaboration coefficients PCF 0.25 and PCT 0.25 . The results of this scenario are shown in Figure 6. The team with a low ability to collaborate is a mismatch with the task that requires high collaboration. Furthermore, results in Figure 6 show that communications technology will not counterbalance that mismatch. Productivity with technology of any TM will be lower than that for face-to- face interactions.

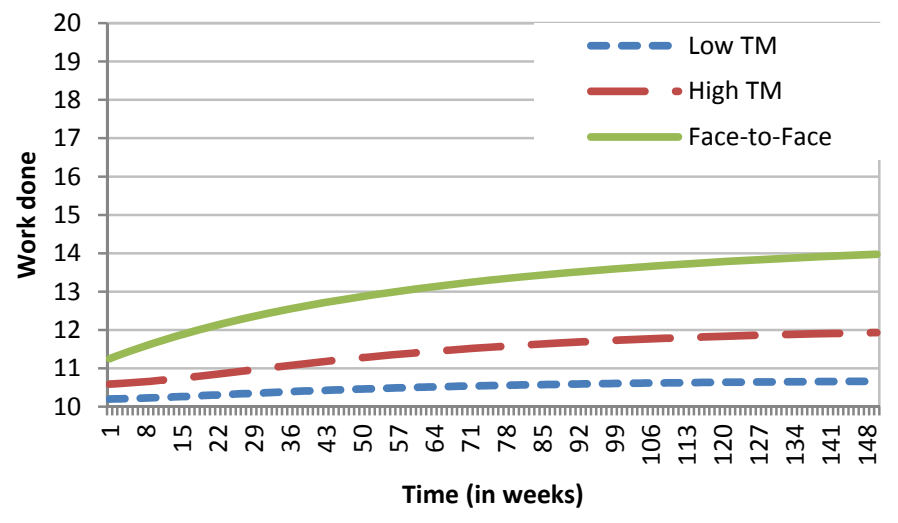

Figure 6: Scenario 3 results

Scenario 4 is where a task requiring high collaboration (ECTask 0.5) is assigned to a homogeneous team with high initial productive collaboration coefficients PCF 0.75 and PCT 0.75 . There is clearly a good match between a team with high ability to collaborate and a task that requires high collaboration. The question is whether team's motivation for the task makes any difference. Figures 7 and 8 show that technology with low TM provides the same productivity as that for face-to-face interaction independent of the team's motivation for the task. However, technology with high TM will have lower productivity with a substantial dip in the early time frame if the team's motivation is low. In that scenario, the team members will be too busy "playing" with novel and intriguing aspects of the technology and their behavior will not adjust fast enough to overcome that loss in productivity due to low motivation. Team with high motivation will adjust quickly its use of the new technology but it appears to take time for its productivity to catch up with that for face-to-face interaction. 


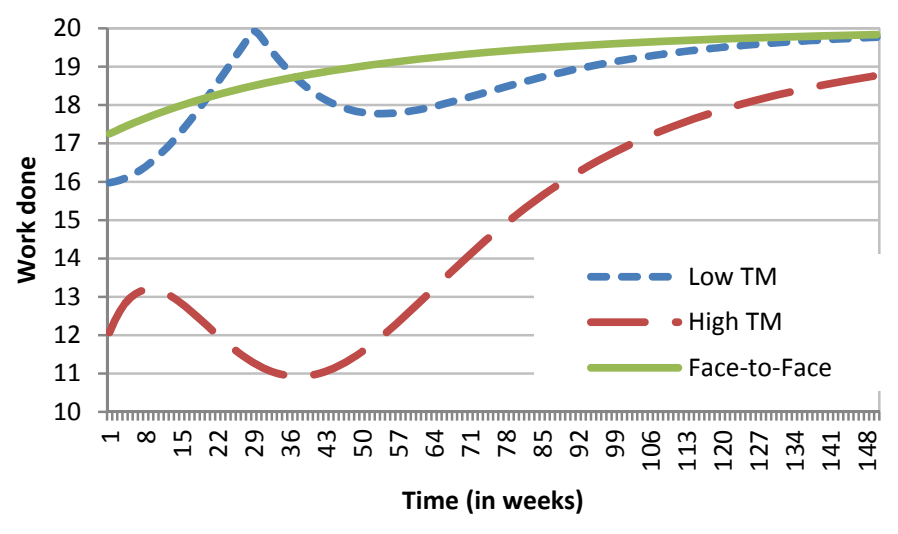

Figure 7: Scenario 4 results for low motivation team

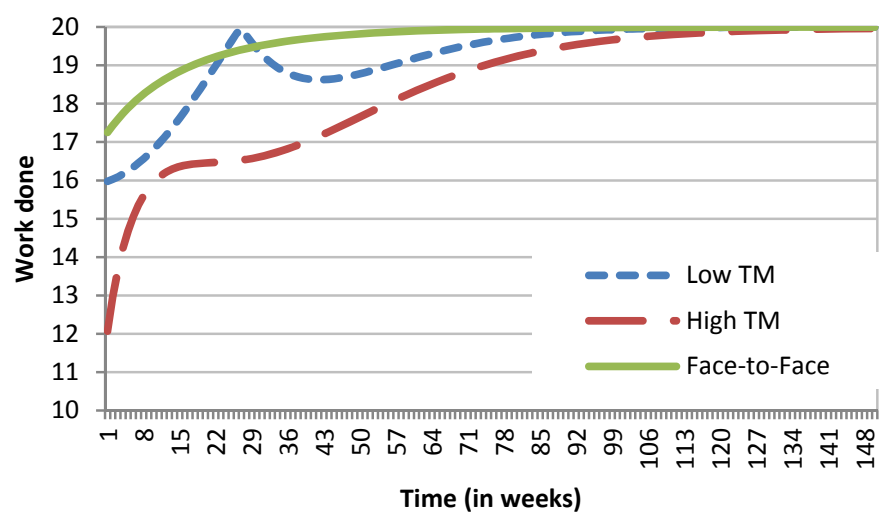

Figure 8: Scenario 4 results for high motivation team

In summary, the results from running the model for the above limited number of scenarios demonstrate the variability in outcomes in the use of communications technologies. Technology with advanced collaboration functionalities (i.e., with high values of TM) may provide productivity for virtual teams that matches or exceeds that of face-to-face interactions. However, the outcome depends on the context. The match or mismatch between the team members' ability to collaborate must match the collaboration required for the task. Furthermore, the motivation levels of the members of the team also play a role in determining any dips in productivity and ability to recover from them. These initial results point to the potential usefulness of the model to investigate the impact of communications technologies on virtual teams in the context of task, team and organizational characteristics.

\section{CONCLUSIONS AND FUTURE WORK}

New and emerging communications technologies with their enhanced collaboration features offer opportunities for virtual teams to carry out their tasks with varying effectiveness as compared to the performance of face-to-face teams performing the same tasks. The results from our initial modeling effort show that the effectiveness depends on the contextual basis of the team and the task. We believe that our model advances our understanding of the complex interactions between communications technologies, teams and tasks, and also provides a useful tool to gain insight into possible outcomes of introducing such new communications technologies. This paper has presented a glimpse into selective scenarios of team and task combinations to highlight some interesting outcomes. We expect that more experimental runs on the model will provide other interesting insight into the team /technology/task interaction complexity. 


\section{Sahasrabudhe, Kanungo, and Iyer}

Areas that have not been explored in any depth in this paper are the role of leadership style, characterization of the emergent states of the teams and potential behavioral impacts on team member characteristics. In addition to refining and enhancing the model for the areas mentioned above, we would like to undertake a detailed calibration and validation of the model with support from practical case analysis. We also plan to add in the model calculations of diffusion of technology. Finally, for the model to be of practical value, we need to define methodologies and tools for mapping the various aspects of communications technology components and features into a TM factor used in the model. Equally important is the need for defining the methodology for analyzing the multiple dimensions of organizational functions and tasks, and mapping them into a task "collaboration index" as defined in the model.

\section{DISCLAIMER}

R. Iyer's affiliation with The MITRE Corporation is provided for identification purposes only, and is not intended to convey or imply MITRE's concurrence with, or support for, the positions, opinions or viewpoints expressed by the author.

\section{REFERENCES}

Balthazard, P., D. Waldman, J. Howell, and L. Atwater. 2004. "Shared Leadership and Group Interaction Styles in Problem Solving Virtual Teams." In Proceedings of Hawaii International Conference on System Sciences.

Burke, C. S., K. C. Stagl, C. Klein, G. F. Goodwin, E. Salas, and S. M. Halpin. 2006. "What Type of Leadership Behaviors Are Functional In Teams? A Meta analysis" Leadership Quarterly 17:288-307.

Canessa, E., and R. L. Riolo. 2006. "An Agent-Based Model of Impact of Computer-Mediated Communication on Organizational Culture and Performance: An Example of the Application of Complex System Analysis Tools to the Study of CIS." Journal of Information Technology 21:272-283.

Curseu, P. L. 2006. "Emergent States in Virtual Teams: A Complex Adaptive Systems Perspective." Journal of Information Technology 21:249-261.

Gartner Group. 2008. "Gartner Says 90 Per Cent of Corporate Virtual World Projects Fail Within 18 Months." Accessed March 30, 2011. http://www.gartner.com/it/page.jsp?id=670507.

Gaston, M. E., and M. des-Jardins. 2005. "Agent-Organized Networks for Dynamic Team Formation.” In Proceedings of 4th International Joint Conference on Autonomous Agents and Multiagent Systems (AAMAS 2005), edited by F. Dignum, V. Dignum, S. Koenig, S. Kraus, M. P. Singh, and M. Wooldridge, 230-237. Utrecht, The Netherlands, July 25-29.

Gautam, D., R. R. Singh, and V. K. Singh. 2009. "Multi-agent based models of social contagion and emergent collective behavior." IAMA.

Goodhue, D. L., and R. L. Thompson. 1995. "Task-technology fit and individual performance." MIS Quarterly 19(2):213- 236.

Guthrie, J. W., M. A. Rosen, and E. Salas. 2007. "The Effects of Collaborative Technologies on Individual and Team Performance in a Network Centric Warfare (NCW) Environment." Air Force Research Laboratory, AFRL-HE-WP-TR-2007-0055.

Hambley, L. A., T. A. O’Neill, and Kline, Theresa J B (2007) "Virtual team leadership: the effects of leadership style and communications medium on team interaction and outcomes" Organizational behavior and human decision processes (103), pages 1-20

Hazy, J. K., and B. F. Tivnan. 2004. "On Building an Organizational Realistic Agent-Based Model of Local Interaction and Emergent Network Structure." In Proceedings of the 2004 Winter Simulation Conference, edited by R. G. Ingalls, M. D. Rossetti, J. S. Smith, and B. A. Peters, 1827-1834. Piscataway, New Jersey: Institute of Electrical and Electronics Engineers, Inc.

Jarvenpaa, S. L., and K. R. Lang. 2005. "Managing the Paradoxes of Mobile Technology." Information Systems Management Fall 2005:7-23 


\section{Sahasrabudhe, Kanungo, and Iyer}

Kozlowski, S. W. J., and D. R. Ilgen. 2006. "Enhancing the Effectiveness of Work Groups and Teams." Association for Psychological Science 7(3):77-124.

Mathieu, J., M. T. Maynard, T. Rapp, and L. Gilson. 2008. "Team Effectiveness 1997-2007: a Review of Recent Achievements and a Glimpse into the Future." Journal of Management 34(3):410-476.

Miller, K. D., and S.-J. Lin. 2010. "Different Truths in Different Worlds." Organization Science 21(1):97114.

Mittleton-Kelly, E. 2003. Complex Systems and Evolutionary Perspectives on Organizations: The Application of Complexity Theory to Organizations. Oxford, UK: Pergamon.

Mollona, E., and G. P. Jesi. 2008. "CoopNet: An Agent-Based Model to Explore Knowledge Integration and Free Riding in Large Organizations." Department of Computer Science, University of Bologna, Technical report UBLCS-2008-09.

Olson, G. M., and J. S. Olson. 2000. "Distance Matters." Human-Computer Interaction 15:139-178.

Stewart, G. L. 2006. "A Meta-Analytic Review of Relationships Between Team Design Features and Team Performance." Journal of Management 32(1):29-54.

Steinfeld, C. (and 13 others). 2001. "New Methods for Studying Global Virtual Teams: Towards a MultiFaceted Approach." In Proceedings of Hawaii International Conference on System Sciences.

Takahashi, S. 2006. "Agent-Based Organizational Cybernetics Approach to Organizational Learning." In Proceedings of SICE-ICASE International Joint Conference, 4591-4595.

Tivnan, B. F. 2005. "Coevolutionary Dynamics and Agent-Based Models in Organizational Science." In Proceedings of the 2005 Winter Simulation Conference, edited by M. E. Kuhl, N. M. Steiger, F. B. Armstrong, and J. A. Joines, 1013-1021. Piscataway, New Jersey: Institute of Electrical and Electronics Engineers, Inc.

Volkoff, O., D. M. Strong, and Elmes, Michael B (2007) “Technological embeddedness and organizational change" Organizational Science 18(5):832-848.

Walsh, J. P., and N. G. Maloney. 2007. "Collaboration Structure, Communications Media, and Problems in Scientific Work Teams." Journal of Computer-Mediated Communication 12(2):712-732.

Zhang, S., J. Fjermestad, and M. Tremaine. 2005. "Leadership Styles in Virtual Team Context: Limitations, Solutions and Propositions." In Proceedings of Hawaii International Conference on System Sciences,.

\section{AUTHOR BIOGRAPHIES}

VIKAS SAHASRABUDHE is an Assistant Professor in the Department of Information Systems and Technology Management, School of Business at The George Washington University. After obtaining $\mathrm{Ph} . \mathrm{D}$. from University of California, Berkeley, he has had extensive practical experience before joining the academics. His research interest is in organizational and management issues in continuing usage of information systems and technologies. His email address is svikas@gwu.edu.

SHIVRAJ KANUNGO is an Associate Professor in the Department of Decision Sciences, School of Business at the George Washington University (GWU). After obtaining his Ph.D. degree (1993) in Information and Decision Systems from The George Washington University he taught at the Indian Institute of Technology at Delhi and since then at GWU. His research interest is in evaluating and assessing information technology (IT) effectiveness in organizations. His email address is kanungo@gwu.edu.

RAMAKRISHNA IYER is an Adjunct Professor in the Department of Information Systems and Technology Management, School of Business at The George Washington University, and is a principal engineer at MITRE Corporation. After obtaining his Ph.D. from Johns Hopkins University, he has had extensive practical experience in voice, data and video communications technologies. His research interest is in organizational and management issues in effective usage of communications technologies. His email address is riyer@gwu.edu. 